INTERNATIONAL HIGHER EDUCATION, Number 72. Summer 2013

Pages 4-5

\title{
Research Collaboration and Global Migration
}

\author{
GALI HALEVI and HENK F. MOED
}

Gali Halevi is at the Informetric Research Group, Elsevier, New York. E-mail: g.halevi@elsevier.com. Henk F. Moed is at the Informetric Research Group, Elsevier, Amsterdam, Netherlands. E-mail: h.moed@elsevier.com. The full text of the article can be found at: http:/ / arxiv.org/ftp/arxiv/ papers / 1212/1212.5194.pdf.

This study compares trends in coauthorship and physical migration of scientists from country to country. Coauthorship analysis has long been used as a way to track the formation of scientific networks both domestically and internationally. Recently, however, an increased interest forms tracking and analyzing authors' affiliations, to follow the physical movement of researchers from one country to another. By analyzing authors' geographical location of a particular paper or studying large sets of articles, international coauthorship and collaboration networks can be identified. Migration, as opposed to coauthorship has an impact, not only on the formation of scientific collaborations but also on the social and economical fabric of a country. Migration trends can, potentially, serve policymakers and programs directors-as to the strengths and weaknesses of their scientific community and whether a country suffers from brain drain or benefits from developments, due to migration. 


\section{Drivers of Migration AND COAUTHORShIP}

Our recent study conducted a multidisciplinary database containing over 20,000 sources of peer reviewed publications, analyzing coauthorship patterns and scientific migration of 17 selected countries-Egypt, Iran, Malaysia, Pakistan, Romania, Portugal, Germany, Italy, Netherlands, the United Kingdom, Brazil, China, India, the United States, Australia, Japan, and Thailand. Analyzing the 2011 corpus of publications and including authors who started their careers from 2001 to 2010, it was able to trace the strengths of immigration between various countries.

The research found a difference between coauthorship and migration patterns. It is apparent that common language and geographical proximity drive international migration more strongly than coauthorships. In addition, the effect of political tensions seems smaller on migration than it is on coauthorship. This can be seen in the relatively low ratio of coauthorship and high migration between Iran and the United States, India, and Pakistan—and China and Taiwan, as examples.

The United States and China are both unique cases of interesting patterns in migration. US authors tend to migrate less frequently than researchers do from large European study countries-United Kingdom, Italy, and Netherlands. This could be due to the sheer size of the United States and the abundance of excellent US research institutions that allows researchers to move from one institute to another without having to leave the United States. In addition, our analysis showed that compared to the level of coauthorship, relatively many young 
researchers currently active in the United States have been previously active in India and Iran.

\section{Permanent vs. Temporary Migration}

Another focus of the analysis was based on the percentages of authors who stay within their country; those who migrate permanently, and those who migrate yet return to the origin country. The largest percentage of authors who stay in their country are American authors, followed by Chinese authors. A much smaller percentage of authors move permanently; and those are from predominantly German and Dutch authors, followed by American and Italian authors. The ones least likely to move permanently are Chinese authors. This could be due to the wealth of resources available to Chinese scientists, as opposed to the lack of expertise. In this respect, Chinese scientists might migrate to other countries, to gain expertise in a certain area, but return to their homeland, to practice and develop their careers. It was also found that the number of authors who migrate and return comprises the smallest percentage of authors. A comparison of the percentages of authors who move permanently to those who move and return to their origin country, a clear picture of countries where brain drain occurs vs. countries which are in the process of developing their infrastructure. Countries such as Iran, Thailand, Malaysia, and Pakistan seem to have a large number of researchers who move abroad and return. This type of migration supports the development of the country's professional-skills levels and infrastructure and shows rising numbers of such exchange. On the other side of the spectrum are countries such as the United States, Japan, India and Germany where larger 
number of researchers seem to be moving to different countries permanently. In the middle, are countries such as China, Brazil, and Australia, where the numbers are balanced between those, who leave their country to work abroad and come back, to those who leave permanently.

\section{Implications on Science Policy}

This analysis, despite tracking existing trends, could potentially serve as a way to examine the effects of migration and collaboration patterns on research performance-especially the extent that researchers who move from one country to another increase their research performance. A case study conducted some years ago on the performance of researchers, at Leiden University in the Netherlands, revealed that those after attaining of their $\mathrm{PhD}$ in the Netherlands conducted their postdoc training at prestigious foreign universities and performed better than those who remained in the Netherlands.

The use of affiliation indicators allows one to track coauthorship patterns and identify the formation of domestic and international scientific networks. Similar use of affiliation indicators have shown that they can be used to track actual physical migration of scientists from country to country, whether on a permanent or temporary basis. This method of analysis enables policymakers at the national level to track researchers who started their career in a country but moved abroad and continued their careers in foreign institutions. This information can play an important role for programs aimed to invite researchers who went abroad to return to their home country. In this manner, one can track migration based also on the scientific focus. If, for example, a country sees 
scientists in neuroscience migrating out, it can decide to invest more in that area, in order to keep its talent and avoid brain drain. This type of analysis can also indicate the formation of centers of excellence around the world. 